\title{
A Study of the Styles of Early Taiwanese Bamboo Chairs According to the Methodology of Style
}

\author{
Shih-Hsing Wu \\ National Yunlin University of Science and Technology, Douliu, Yunlin, Taiwan \\ Ying-Pin Cheng \\ Hsuan Chuang University, Hsinchu City, Taiwan \\ Chi-Hsiung Chen \\ National Yunlin University of Science and Technology, Douliu, Yunlin, Taiwan
}

\begin{abstract}
Handicrafts and furniture of bamboo once flourished in Taiwan during the 1970s and 80s but have since gradually declined after that period of time. The viability of the handicrafts industry was greatly threatened along with there being a real possibility of losing this traditional heritage. Nowadays, green products are a growing trend in the fashion industry, and thus some Asian countries have realized the importance of using green products and its development of cultural creative assets. The literature/study of the styles of traditional Taiwan bamboo arts are rare but they are worth exploring. Therefore, the purposes of this study are as follows: (1) to explore the category of traditional Taiwanese bamboo column furniture; and (2) to explore the backgrounds, forms, and contents of traditional Taiwanese bamboo column furniture. The results show that: (1) traditional Taiwanese bamboo chairs can be divided into eight categories: stool, side chair, slant-back reclining chair, tai-shi armchair, pair of gong-po chairs, two-seat chairs, "mother \& child” chairs, and other styles of chairs; and (2) traditional Taiwanese bamboo column chairs styles originate from early Chinese Fujian and Guangdong provinces, and these bamboo chairs' forms have imitated the wood furniture of the Ming and Qing dynasty furniture. However, the technology of the enclosed-pipe structure is a special handicraft skill that reflects the early Taiwanese' resourcefulness in farming and fishing. We hope this paper can promote relevant and practical foundation research and studies.
\end{abstract}

Keyword: style, bamboo furniture, cultural identity industry, handicraft

\section{Introduction}

The town of Zhushan can be regarded as the main area for the design, creation, production, and sales of Taiwanese bamboo craft and furniture. When the Taiwanese economy took off in the 70s-80s, there were approximately 2,000 bamboo craft factories flourishing in this area. Alas, they have subsided with the passing of the years (Wu, Cheng, \& Chen, 2005).

Shih-Hsing Wu, Ph.D., bamboo glass workshop Learning Centres Chairman, Graduate School of Design, Doctoral Program, National Yunlin University of Science and Technology.

Ying-Pin Cheng, assistant professor, Department of Fashion Design, Hsuan Chuang University.

Chi-Hsiung Chen, Ph.D., professor, Department of Creative Design, National Yunlin University of Science and Technology. 
Bamboo furniture can be treated as one of the specialty products unique to Taiwan. However, the dying out of older generation traditional bamboo furniture artisans, coupled with the lack of attraction to the younger learners in picking up the craftsmanship through vocational education, has brought on realistic worry about the serious discontinuity in the passing on of this distinctly unique Taiwanese cultural industry (Lee, 2000; Wu, Cheng, \& Chen, 2004).

In response to the high regard for environmental and green products in today's industrial design world (Ehrenfeld, 1997), the bamboo-producing countries have all actively manipulated bamboo to develop as green products in recent years (Chung \& Yu, 2002). Bamboo grows speedily (Chung \& Yu, 2002; Ghavami, 2005), is environmentally friendly and allows diverse usages (West \& Louys, 2007). Therefore, this opens up new business opportunities, but the impact cast by the materials used is also a concern (Bovea \& Vidal, 2004). The current available bamboo furniture is still relatively rough. Some bamboo furniture from Asian countries has inherited the traditional look, yet its low durability is a drawback that cannot fit the lifestyles of modern customers. Today, some Asian countries are conscious of the important roles of cultural and creative industries in future economic developments (Yusuf \& Nabeshima, 2005). This has lead to the revival of the Taiwanese bamboo furniture industry which once prospered but that was now also on the verge of extinction.

How can we get a grasp on the traditional style of bamboo craft in Taiwan when studying bamboo furniture and other daily amenities? Though we may be able to get some ideas through 20th Century Taiwanese Bamboo Crafts by Lee (2000), the book cannot provide a thorough idea as we explore things further. Since there is an array of Taiwanese traditional bamboo, in order to facilitate the study of Taiwan's traditional style of bamboo amenities, this paper shall specifically focus on the study of chairs and other types of furniture made out of bamboo.

Based on the aforementioned research background and motivation, this paper sets the research goals to be as follows: (1) to study the types of Taiwan's traditional bamboo furniture; and (2) to study the background style, form and content of Taiwan's traditional bamboo chairs.

This study applies the research techniques of content analysis of the documents used in this multi-case study. The resources of the case studies come from the pictures of bamboo furniture from "The Bamboo Daily Amenities of Taiwanese residents in the 20th century” by Lee (2000) with the photos being filed for this study. The collections and analysis of images are used in the techniques in analyzing data. Through literary review, comparisons of pictures, and written materials, this study tries to dissect the style of Taiwanese traditional bamboo furniture.

\section{Literature Review}

\section{Taiwanese Bamboo Furniture}

In 1943, Japanese folk crafts expert, Zong-yue Liu launched an investigative study in Taiwan. When he saw the austerity of bamboo furniture, he was deeply joyed by its charm. He believed that the bamboo furniture, displayed the beauty of the material and the uniqueness in structural design. He therefore held Taiwanese traditional bamboo craft in high esteem (Lee, 2000).

As a matter of fact, bamboo was an indispensable material in ancient Taiwan. Ya-tang Lian (1878-1936) considered that Taiwanese bamboo craft experts can make beds, coffee-tables, small and big baskets, as well as daily amenities out of the bamboo, in The General History of Taiwan ( published in 1918) (Lee, 2000). Back then, 
the weaving of slender bamboo strips was the part-time job of peasant women in their free time. The making of big-scale furniture that involved chiseling had needed the men's craftsmanship. The bamboo craft works have gradually converted from traditional Fujian and Cantonese styles into Taiwan's very own featured style (Lee, 2000).

According to current data, there were about three primary categories of bamboo furniture that were used in early historical Taiwan (Lee, 2000, Wu, Cheng, \& Chen, 2004, Wu, et al., 2005): (1) Drawing from Fujian and Cantonese styles of the Ming dynasty, artisans were able to make furniture such as armchairs (for scholars/bureaucrats), wash stands, flower stands, bambusa stenostachya shelves, reclining chairs, and bamboo stools; (2) The daily usage style can be seen in bamboo tables, bamboo chairs, bamboo beds, bamboo stools, children's chairs, strollers, bamboo cabinets, and shelves; And (3) creative and artistic styles consisted of crown chairs, mandarin duck — shaped pillows and strollers. In view of the design style, the two former categories mainly take on the traditional forms used in wooden furniture. The third type is modified furniture integrated with a modern design concept. In regards to the decorative concept, the three aforementioned categories apply heat to bend the slender pieces of bamboo, and the carving of the bamboo, had partial usage of certain mixed materials such as glass, rattan, metals, and plastics as embellishments to the form (Wu, et al., 2005).

Traditional bamboo furniture tends to imitate the forms used in wooden furniture. Yet bamboo is hollow and has no xylem. Its fiber and stem are hard and grow in parallel form, resulting in the unusual bamboo furniture production methods that differ from those of wooden furniture. The major production methods of bamboo furniture are: gouging, chiseling, paper-cutting, stem-enclosing, and heating to bend bamboo, fixing the bamboo dowels, splitting bamboo, seat-top making, cabinet corner gussets, mortise-and-tenon-joints, round frame making, and decorative bamboo floral patterns, etc.. Among which, the stem-enclosing technique (see Figure 1) is the core technique in Taiwan bamboo furniture-making. This is a special production technique used in bamboo work. It encompasses the aesthetics and decorative functions by using just one bamboo stem (see Figure 2) as the main structure, added with drilled holes and the enclosure of other structures to form a tightly integrated structure. All limbs are often applied during production (see Figure 3) (Wu, et al., 2005).

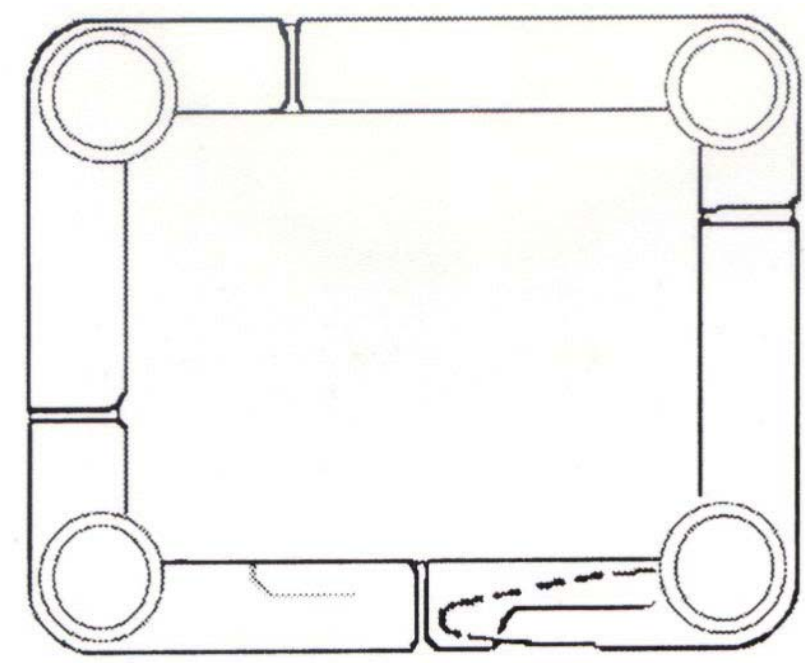

Figure 1. Stem-enclosing technique, Schematic plan view. 


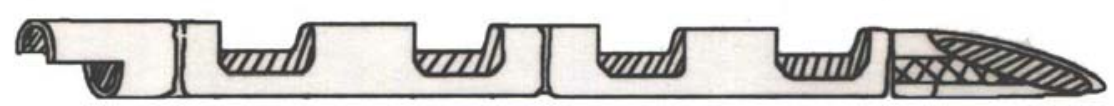

Figure 2. The Holes and the Front and Rear of the Stem.

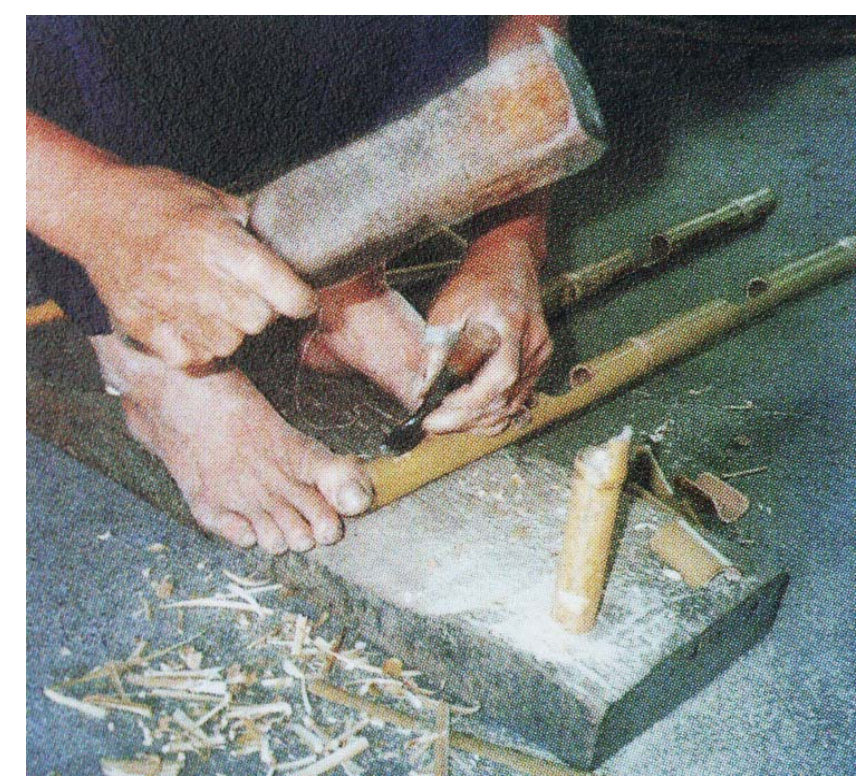

Figure 3. All limbs applied during the production process.

The weaving of bamboo leaves is also one of the more significant techniques in bamboo furniture. First the bamboo stems are sliced into many thin strips and woven into surface that can be used as a seat or table top (see Figure 4). During the production process, a welding torch is required to heat and bend the bamboo. Strings are tied to the bamboo temporarily to secure the shape, and will only be untied once the connections between the stems are completed (Lee, 2000; Lin, 2002; Wu, et al., 2004; Wu, et al., 2005).

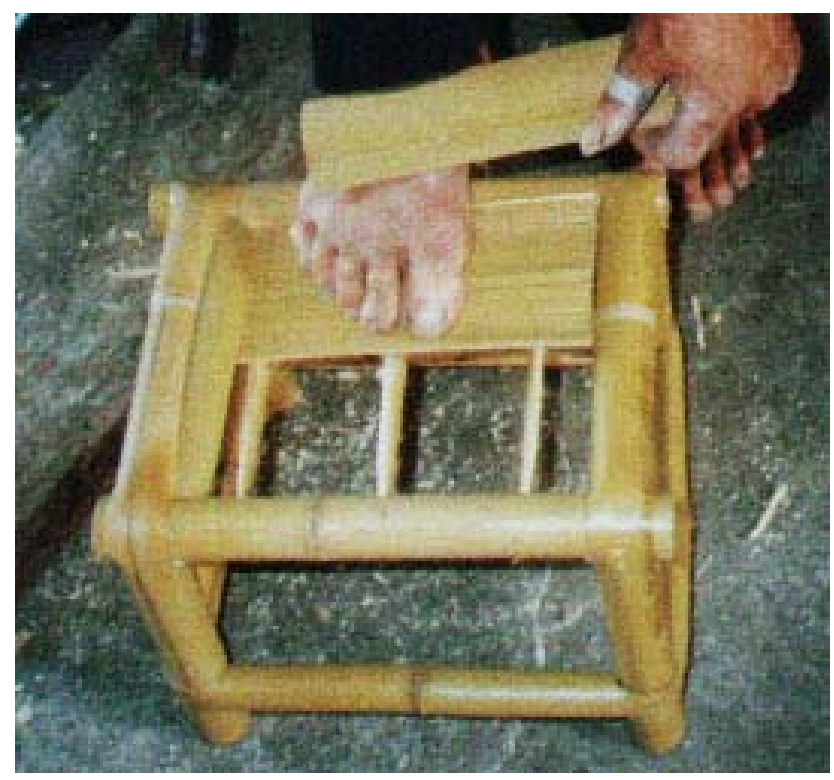

Figure 4. Weaving bamboo strips into a seat top. 
In the past 20 years, the Taiwanese civilian factories and the National Taiwan Craft Research and Development Institute have demonstrated substantial progress in the design and development of different bamboo furniture forms. This has fostered the plastic art of modern bamboo furniture, which branches out into two categories of design and production, namely being the "bamboo furniture in its natural form" and "laminated bamboo furniture” (Lee, 2000; Wu, et al., 2004; Wu, et al., 2005). (1) Bamboo furniture in its natural form (see Figure 5): usually maintains the traditional production process, which shows connections between stems of bamboo, and conveys a plain and artless style; And (2) the laminated bamboo furniture design (see Figure 6): bamboo material that has been processed in order to become laminated boards can therefore be more easily used in creating the forms like those of wooden furniture. Composite materials can be used together, so there is higher flexibility and plasticity in form design. As laminated bamboo furniture allows for easier innovation and carries the bamboo's texture, thus it is becoming more widely used.

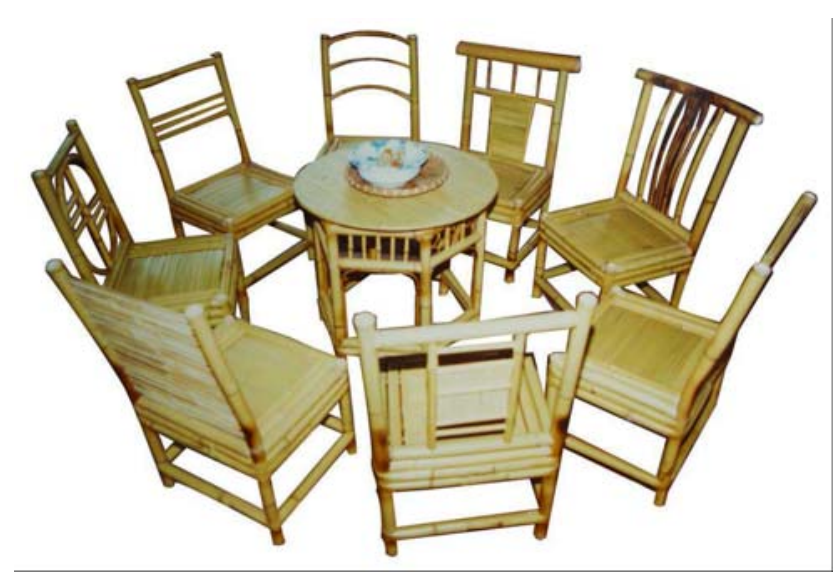

Figure 5. Bamboo Furniture in its natural form.

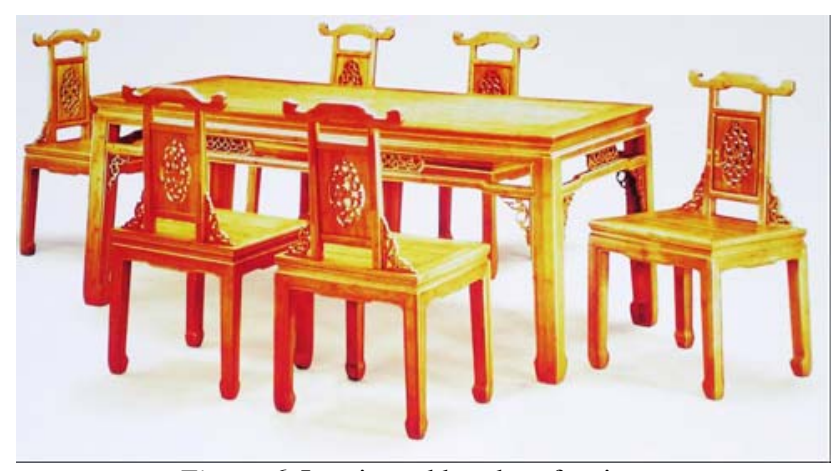

Figure 6. Laminated bamboo furniture.

Overall, Taiwan bamboo furniture shows the following characteristics and trends (Wu, et al., 2005): (1) There are totally different production methods for traditional and laminated bamboo furniture used by today's bamboo furniture factories. The former one is plain, neat, and low-cost. The latter one is luxurious, natural, and expensive; (2) The traditional method of bamboo furniture making uses traditional production techniques, but laminated bamboo furniture is made by using carpentry techniques; And (3) this means that it is not easy to create new forms of traditional bamboo furniture as it needs to deal with the bamboo's physical property first and foremost. The laminated bamboo furniture however is made by using carpentry techniques. This results in there 
being no differentiation between bamboo furniture and wooden furniture, a point worthy of further contemplation.

Therefore, in not only keeping with the traditional features, traditional bamboo furniture also needs to strengthen its production techniques by copying carpentry techniques.

Moreover, carpentry production techniques can be combined with traditional bamboo production techniques in producing laminated bamboo furniture so as to retain the spirit of conventional culture and the characteristics of natural bamboo. Lastly, besides working with bamboo in more traditional methods, designers also need to study the materials itself to find out new possibilities of use. When working out a creative design in bamboo furniture, we also need to understand the characteristics and styles of Taiwanese traditional bamboo furniture. This means passing on a heritage and looking forward towards future developments of the craft.

\section{Style}

The definition and explanation of style differs according to the time period, history, and local geographic region. According to the explanation of the English Cobuild Dictionary, Harper Collins (2003): (1) The style of something is the general way in which something is made or presented, which often shows the attitudes of the people involved in the making of the respective furniture; (2) If people or places have style, then they would be smart and elegant; (3) The style of a product is its design; And (4) in the arts, a particular style is characteristic of a particular period or group of people.

The Oxford Advanced Learner's English-Chinese Dictionary (Hornby, 1996) states the meaning of style as (1) the different types of writing or language; (2) a unique type of an artist, a writer or someone working in other forms of the arts; (3) the way or style of behavior; (4) a representation of the refined taste of somebody or something; and (5) the style or design of fashions. From the explanations of style stated in the aforementioned dictionaries, we can tell that the word style has several different meanings.

Ross (1982) explicates the concept of style as (1) the style of an organization, or school of thought; and (2) an individual's ideology in style. Ross believes that style is the study of the similarities and differences among various kinds of works, which means the generalization and uniqueness of style. Individual works of art will be presented in style in relations to their diverse and complex aesthetics. That is exactly what the fundamental value of art and design is.

In Chen \& Cheng's (2005) discussion of the theory of style, they argued that "background" and "constitution" were the two major factors that formulate style. "The background of the style" as a factor refers to is: (1) the implied properties of time, i.e., within the same era, a period of time as in years, or the beginning period of time, etc.; and (2) the implied properties of space i.e., the environment, country, society, school of thought, company, and organization, etc..

While "The constitution of style" refers to: (1) the implied properties of form, i.e., the original entity, and the appearance of the material, its technique/technology, the consistency, the paradigm, symbols, features, influences, trends, its new definitions/declarations, the things that are inherent about it, and the sense of differentiation, etc.; and (2) the implied properties of "content” are its value, ideology, history/culture, strength, ideas, missions, the norms, the people's attitude, aesthetics/tastes, philosophy, vision/anticipation, creativity, meaning, and the process of solving problems, etc.. 
In reviewing the literature on style, this paper deems that there can be a simplification of the idea that would constitute the implied properties of style that are proposed by Chen and Cheng (2005) into three constituting elements—-the background, its form, and content, with an omission of the implied properties in each constitution (see Table 1).

Background-environments, regions (nations), the inheritance, and its organization; style-form, the technique, symbol, and differentiation; content—value, ideology, culture, idea, taste, creativity, and meaning. It is hoped that the constitution and implied properties that formulate style can help artisans or the study of the style of craft works.

Table 1

The Constituting Factors and Implied Properties of Style

\begin{tabular}{|l|l|}
\hline $\begin{array}{l}\text { The constituting factors and implied properties of style } \\
\text { Constituting Elements }\end{array}$ & Implied properties \\
\hline Background & Environment, region(nation), inheritance, organizations \\
\hline Style & Form, technique, symbol, differentiation \\
\hline Content & Value, ideology, culture, idea \\
\hline
\end{tabular}

\section{Methods}

To achieve the goals of this study, this paper applies research techniques of content analysis of the documents used in the multi-case study. Since there is an array of Taiwanese traditional bamboo, in order to facilitate the study of Taiwan's traditional style of bamboo amenities, this paper specifically focuses on the study of the chairs of Taiwane bamboo furniture. The categorization of chairs is based on "The Bamboo Daily Amenities of Taiwanese residents in the 20th century” by Lee (2000). It lists the following: (1) bamboo stools; (2) side chairs; (3) reclining chairs; (4) armchairs (scholar-bureaucrat); (5) couple chairs; (6) double seaters; (7) children chairs; and (8) others. Other daily amenities such as coffee tables, bamboo screens, bamboo beds, and cabinets are not covered in this study.

During this study, the bamboo chairs listed in "20th Century Taiwanese Bamboo Crafts" by Lee are used as case studies. We took pictures of the bamboo chairs, and had filed for individual and detailed dissection comparisons. This was to find out the similarities and differences for further study and the inferences on the analysis of the results are shown below.

\section{Results and Discussion}

\section{Categorization and Introduction of Case Studies}

(1) Bamboo stool (see Figure 7)—roughly grouped into bambusa stenostachya and makino bamboo stools. The stem body of bambusa stenostachya is thick, sturdy, and cannot be bent easily. Carpentry production techniques are mainly used in working on this kind of bamboo. Makino bamboo is soft, bright in tone and elastic, and has a pleasant tactility and visual effect that carries a refreshing and natural sensation.

(2) Side chairs (see Figure 8 \& Figure 9)—Regular chairs used by residents in farming villages in Taiwan, usually made from makino bamboo, are either armed or without arms, the production methods used are the same as of a bamboo stool. Those without arms are composed of simple lines, sharp, and clear designs. Armed chairs are shorter, lighter, and have a wider seat surface. 
(3) Reclining chairs (see Figure 10 \& Figure 11) - There are two types of reclining chairs: dual- functioned and fix-functioned with extensibility. Adjustable chairs allow for a recumbent position, making it less rigid as armchairs, and therefore can be regarded as the best seat for resting. Fix-functioned chairs are mainly for lying down, with an additional chair used as foot rest. In early times, residents often used them while taking naps in the afternoon.

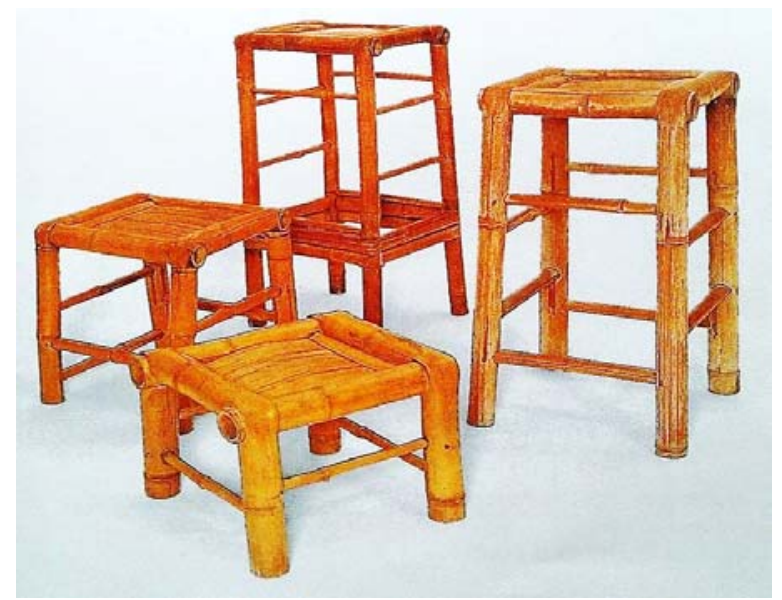

Figure 7. Bamboo stool.

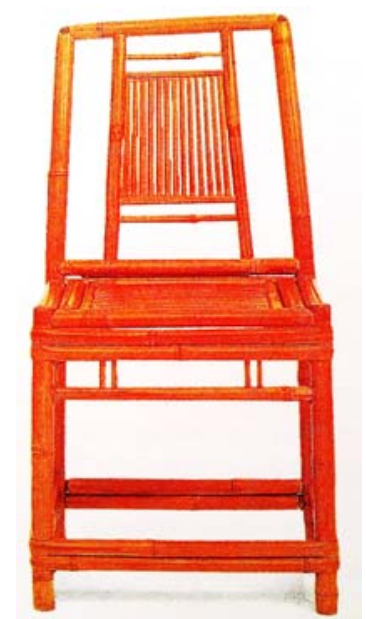

Figure 8. Armless side chair.

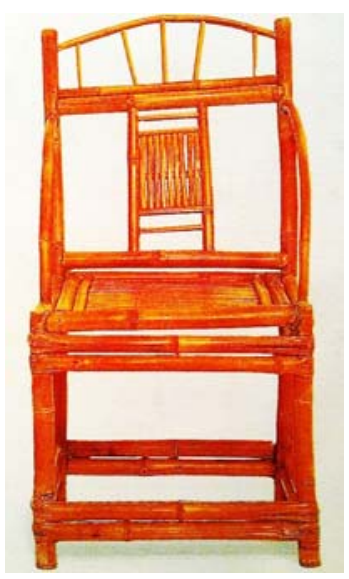

Figure 9. Armed chair. 


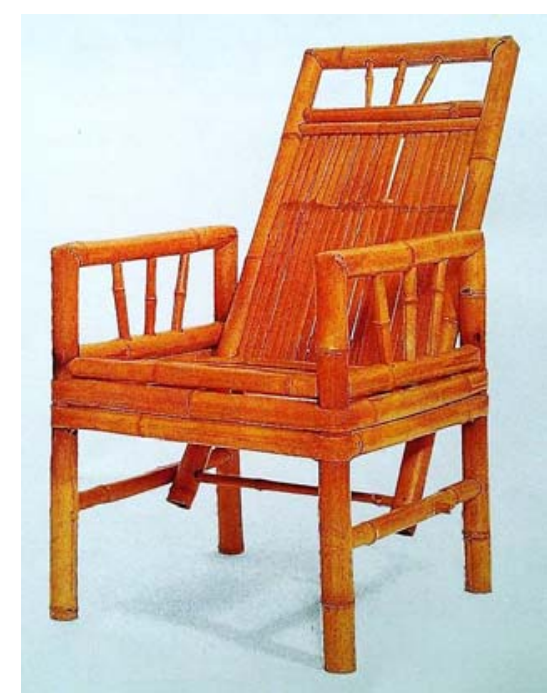

Figure 10. Dual-function reclining chairs.

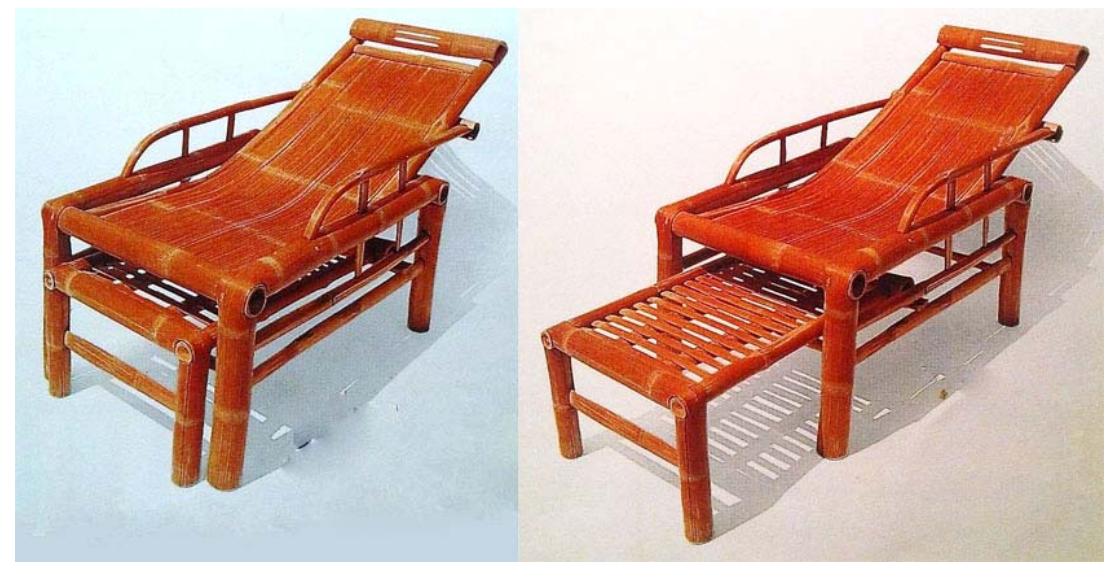

Figure 11. Fix-functioned chair with extensibility.

Chiseling and gouging at special angles and heat-bending are the main production techniques used to work on this kind of bamboo. Mechanical tools cannot be used to replace these kinds of techniques. The bamboo chairs are distinctively characterized by their being easy to carry and comfortably cool for sitting on.

(4) The armchair (for scholars/bureaucrats) (see Figure 12 \& Figure 13)—Possesses China's Fujian and Cantonese traditional forms, that are grouped into two styles-plain or grand. Plain ones are usually for guests and the elderly. The grand style ones are made from selected bamboo materials, and are then painted, and backed with an inlaid piece of red and blue decorative ceramics. Both styles come with heat-bent bamboo ornaments and ornaments in $\mathrm{H}$ or $\mathrm{S}$ shapes. The chair's bamboo sectors are neatly and delicately treated, demonstrating a natural style, and are not to be owned by the general public. Ming-jia Cheng, an artisan from the town of Lun-bei, in Yun-lin county, had inherited bamboo furniture making techniques from older generations that were passed onto him, and is known for his production of armchairs (for scholars/bureaucrats). The Eight Trigram form of the chairs display refined production techniques, with the back and arms being both inlaid with heat-bent slender bamboo, floral decorations, each of which carries some customary meanings, and are stylish and full of aesthetic beauty.

(5) Couples chair (see Figure 14) — Placed in the bedroom, they are made armless probably for saving space and making conversation between a happy couple easier. They are designed like side chairs but appear in pairs. 


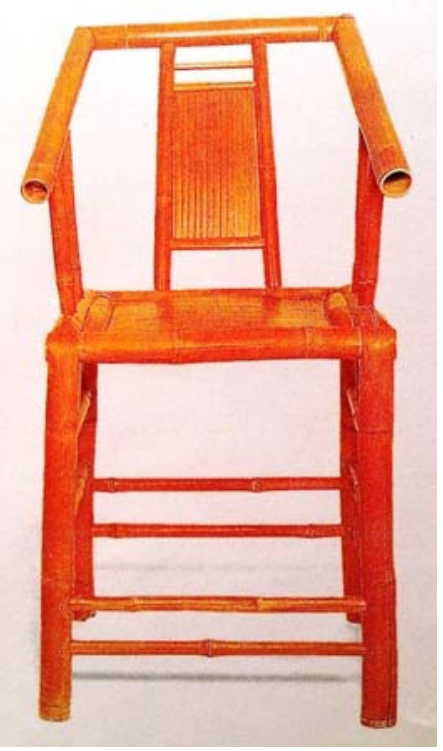

Figure 12. Plain armchair.

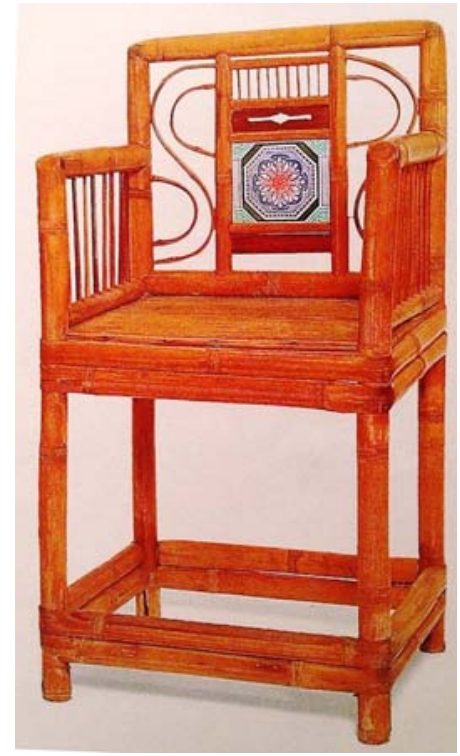

Figure 13. Grand armchair.

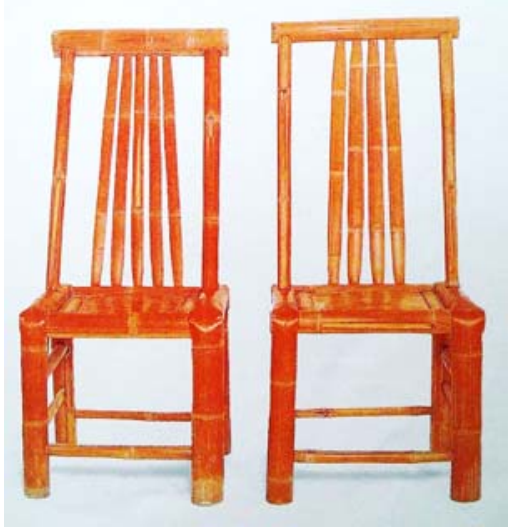

Figure 14. Couple chairs. 
(6) Double seaters (see Figure 15) - These are an extension of the single seat chair, and are probably good for increasing space for sitting on while it reduces the amount of production material and time spent creating it.

(7) Children's Chair (see Figure 16)—Mainly, they are the infant chairs. If inverted, it can be sat on by a nanny.

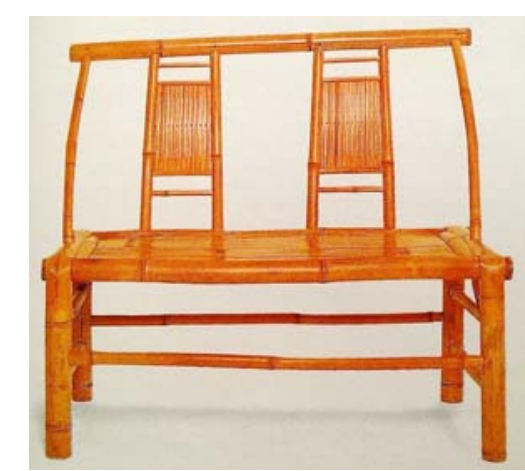

Figure 15. Double seat.

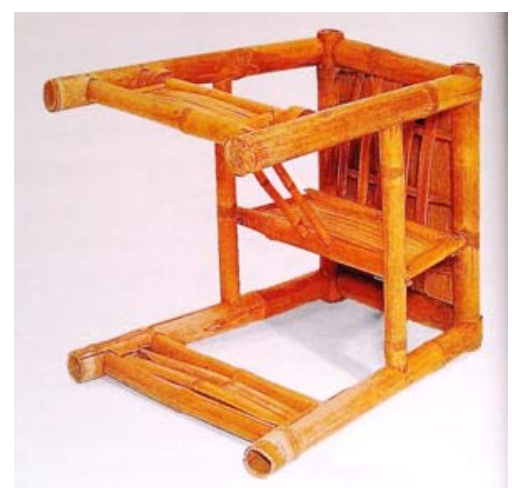

Figure 16. Children's chair.

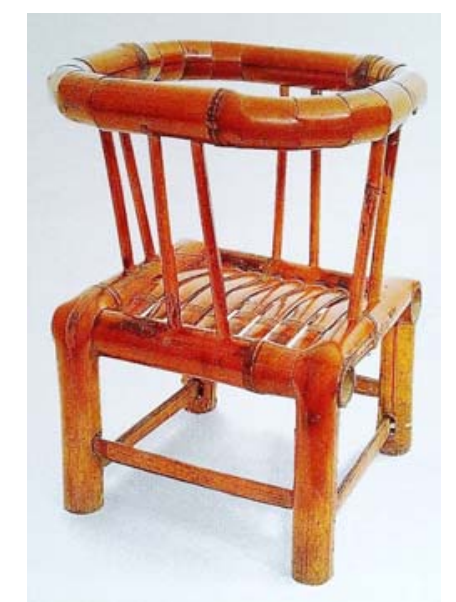

Figure 17. Sedan.

(8) Others (see Figure 17)—These are called sedans in The Bamboo Daily Amenities of Taiwanese residents in the 20th Century by Zan-shou $\mathrm{Li}$ (2000). Li only mentioned that it can be sat on from many directions but he did not convey the explicit function of the sedan. However, it can be used as an infant chair that is fit for tying onto the back of an adult who then can work and take care of their baby at the same time. 


\section{The Style Analysis of Case Studies}

This study uses the constituting elements and implied properties of style that are shown in table 1, combined with the results of literature reviews, to conduct analysis on each item of the implied properties of styles in Taiwanese bamboo furniture (see Table 2).

Table 2

The Constituting Elements and Implied Properties of Style

\begin{tabular}{|c|c|c|}
\hline Constituting Elements & Implied Properties & Explanations \\
\hline \multirow[t]{3}{*}{ Background } & Environment & Subtropical zone, island, bamboo-producing \\
\hline & Region & $\begin{array}{l}\text { Farming and Fishing villages in early } \\
\text { Taiwan }\end{array}$ \\
\hline & Inheritance & Chinese cultures from Fujian, Canton \\
\hline Style & $\begin{array}{l}\text { Form } \\
\text { Technique } \\
\text { Symbol } \\
\text { differentiation }\end{array}$ & $\begin{array}{l}\text { See Figures } 8,9, \& 18 \\
\text { See Figures } 19, \& 20 \\
\text { See Figure } 21 \\
\text { See Figure } 22\end{array}$ \\
\hline Content & $\begin{array}{l}\text { Value } \\
\text { Ideology } \\
\text { Culture } \\
\text { Idea } \\
\text { Aesthetics } \\
\text { Creativity } \\
\text { Meaning }\end{array}$ & $\begin{array}{l}\text { Practicality and Admiration } \\
\text { Accumulation of life's experience } \\
\text { Taiwanese agricultural culture } \\
\text { Wisdom of living and the application of handicraft skills } \\
-- \\
\text { Using bamboo to imitate wooden furniture } \\
\text { Practical utensils in simple life }\end{array}$ \\
\hline
\end{tabular}

Source of data: compiled in this study.

From the Table 2, we can know the background factors of the different styles of Taiwanese bamboo furniture which, mainly inherits from China's Fujian and Cantonese cultural traits of the past. Taiwan, in early times, was still fishing and farming society. As an island with limited resources, and poor living standards but having a proficient supply of bamboo, the island's artisans had developed bamboo into furniture to be made for daily use. The styles of furniture and their implied content carried both practical, trustworthy, and admirable values, displaying a wisdom of living and the application of skills of handicrafts. This had also demonstrated an accumulation of living experiences and with no doubt, that was an imitation of Ming and Qing dynasty wooden furniture designs.

Below is the analysis and explanations of the styles of Taiwanese bamboo furniture:

(1) Form — tends to imitate the Ming and Qing dynasty furniture styles. First the form of a bamboo stool is made based on a square surface with increased height (see Figure 18). It is closely related to ergonomics, users, situational and functional purposes. Being something that is easy to carry and that can be produced quickly also has turned it to become the most widely used daily amenity. To meet the situational and home decoration functions, the back and arms are added to the bamboo stool making it a side chair with back and arms (see Figure 8 \& Figure 9).

(2) Techniques-Stem-enclosure is the core technique in Taiwanese bamboo furniture-making. It is featured by using one's choice of bamboo as the main structure, added with chiseled holes made at 45 degrees and uses the enclosing of other structures to form a tightly integrated structure. It is a technique that encompasses aesthetics and decorative functions. There are two types of stem-enclosure. The vertical one is for enclosing the chair legs whereas the horizontal one is for enclosing a bamboo mat (see Figure 19). 


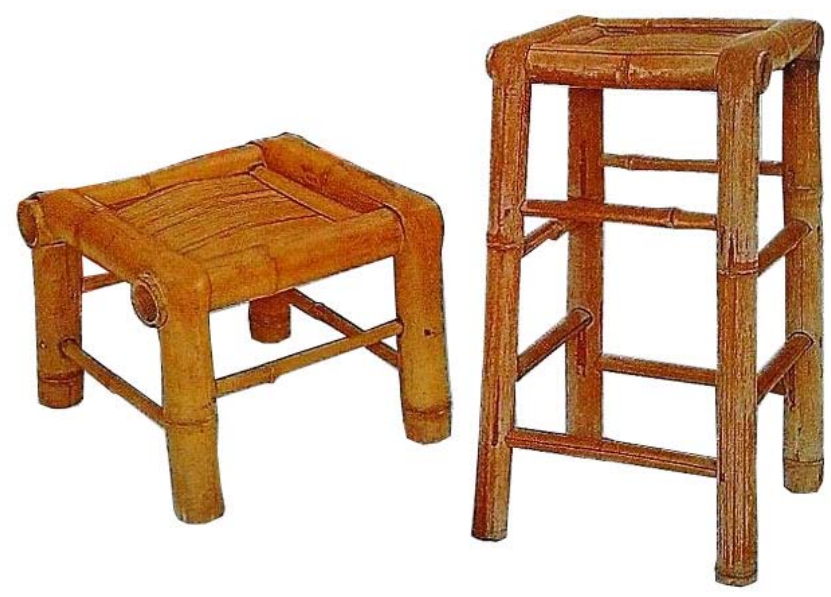

Figure 18. A stool developed from a square surface with increased heig.

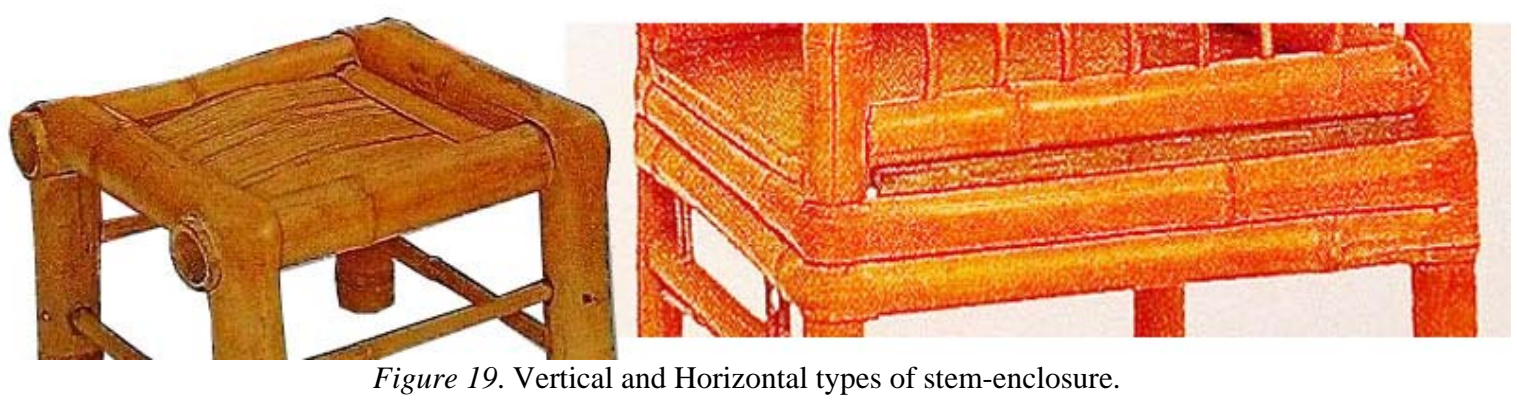

In addition, weaving of the bamboo mat requires the technique of splitting up a huge amount of bamboo strips from the vein of the bamboo stem. The loosely connected bamboo strips will be laid flat, and inserted into the bamboo pins at both ends to make the seat, table top or bed surface. This production process requires fire spray to heat-bend the bamboo stem which temporarily fixes the problem of the strings hence avoiding the bouncing back or coming off of any parts. As there is a sequence in the assembly of parts, the artisan usually needs to use all his limbs in the production process. Taiwanese bamboo furniture in its natural form is much more restricted in its form as the bamboo needs to be especially bent, so that they usually display a square-looking structure with insertions. For decorations, the heated slender bamboo strips are often introduced to soften the look of the form and thus making the furniture more delightful and pleasing to the eyes (see Figure 20).

(3) Symbols-The rectangular forms of the back and chair legs are imitations of Ming dynasty furniture (see Figure 21). This reveals the origin and inheritance of modern Taiwanese bamboo furniture. The decorations here are often presented in strict lines through bamboo weaving, and they are also very common in the chairs that have a comb-like back such as with Ming dynasty furniture.
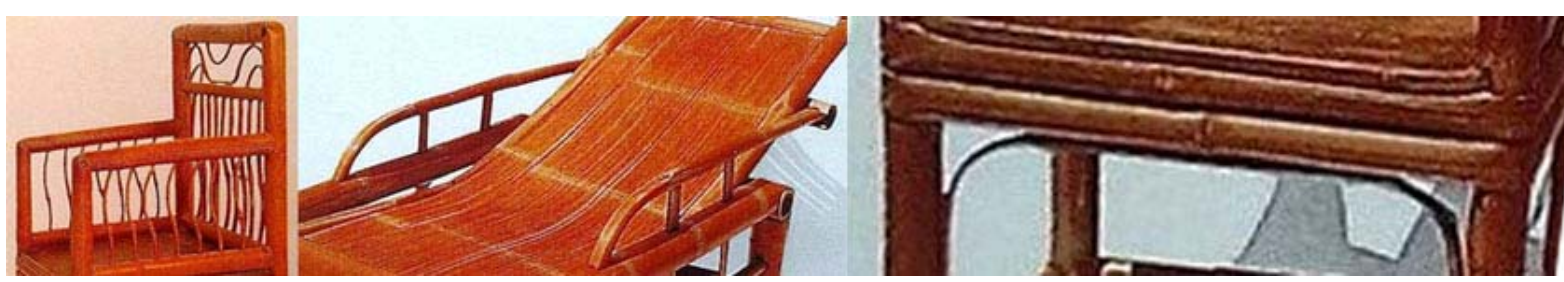

Figure 20. The decorative effects presented by the applications of curvature through the usage of slander bamboo strips. 


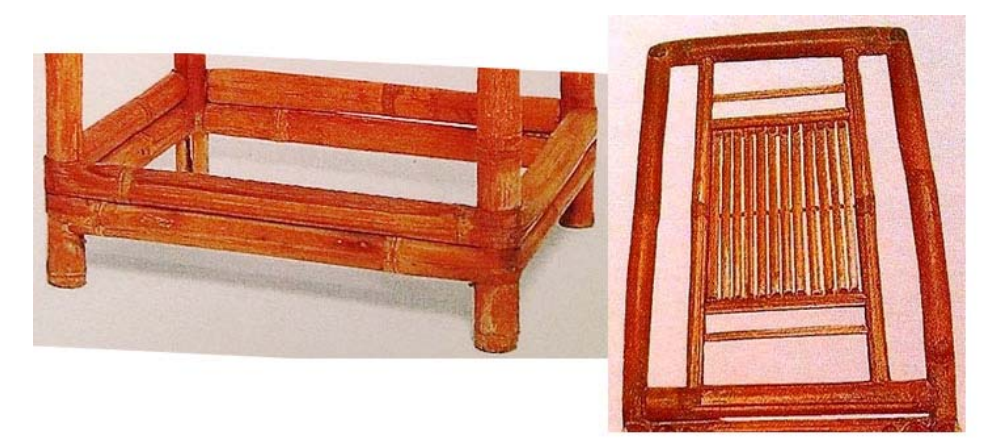

Figure 21. The rectangular form of the back and chair legs are imitations of Ming dynasty furniture.
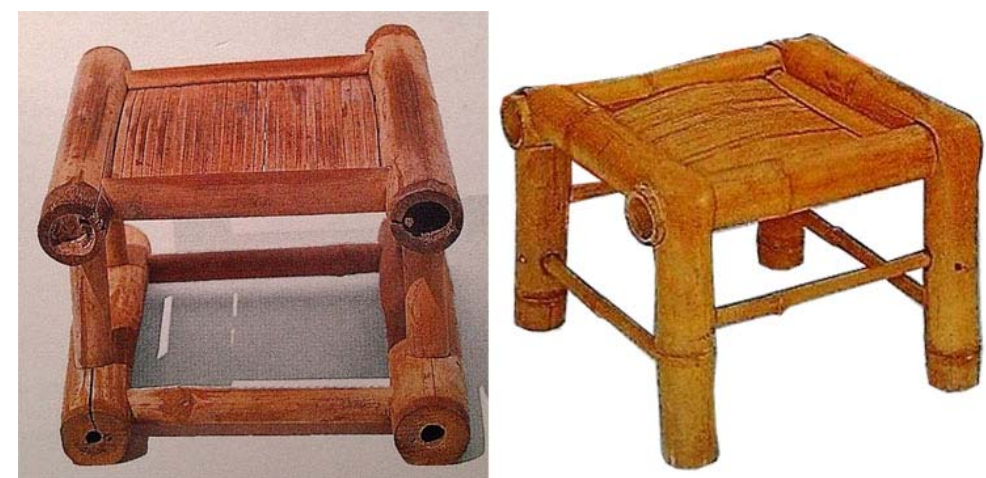

Figure 22. The different structure between non-stem-enclosure and stem-enclosure.

(4) Differentiation-We can judge whether a piece of bamboo furniture is made by the traditional Taiwanese bamboo furniture style by examining if it has stem-enclosed structures (see Figure 19) and back decorations (see Figure 13). Most of the traditional Taiwanese bamboo furniture will be made with stem-enclosing techniques to reinforce the chair's structure while making it more pleasant in its appearance. The furniture with its enclosed stem usually appears smooth and warm, pleasant to the touch and ergonomic. Chairs without stem-enclosed parts appear rough and overly simplistic in structure (see Figure 22), making the form very crude. Traditional Taiwanese farming societies usually were minimalistic, but friendly, polite, and modest. Therefore, one's household furniture should also better reflect this sense of simplistic harmony.

\section{General Discussions}

Through the style analysis of the case studies quoted above, we know that most designs are intimations of Ming and Qing dynasty furniture, particularly the Ming furniture. In fact, considering everything from the point of view of practical living experiences and cultural systems, it is understandable why bamboo has been adopted to produce chairs that can be seen daily and that are used often. The bamboo stem used in furniture and the wood used in Ming dynasty furniture share a commonality in roundness. The origin of bamboo chairs can date back to Song Dynasty when literati paintings deeply influenced the Ming dynasty furniture. Therefore, it makes sense for artisans of traditional Taiwanese bamboo furniture to make imitations of Ming dynasty furniture.

In regards to the techniques, the stem-enclosing, the taking of measurements, heat-bending, and floral patterns are the most prominent features in Taiwanese bamboo furniture. We cannot trace these techniques back to the original source, but maybe we can explore this through ancient Chinese paintings. However, as seen from the collected pictures, most chairs do not show the usage of these techniques. The stem-enclosing technique 
makes the bamboo chairs fitting and sturdy. In fact, it is well known that cutting hollow and round structures from bamboo is troublesome, so typical bamboo furniture is usually stem-enclosed. Yet traditional Taiwanese bamboo furniture does not apply this technique when cutting the stem. Artisans must select straight and thick stems, with suitable nodes for production, so that the stems can be well treated in its joints and bent parts. It is inferred that the appearance of the stem-enclosing technique is used to reduce the number of times one uses to cut off the stem, for structural reinforcement, and durability.

Moreover, stem-enclosure also produces a soft tactility and a texture that does not hurt people's skin. Therefore, this technique is mostly used for the seat's surface, the joints of chair legs, and the underside of the mat and the fixing of chair legs. These parts in fact are the most protruding parts of a chair and are most frequently in touch with human body parts. In 1943, Japanese folk crafts expert, Zong-yue Liu launched an investigative study in Taiwan. When he saw the beauty of the material and uniqueness of the structural design in bamboo furniture, he was deeply joyed by its charm and therefore gave it his high commendation (Lee, 2000). Although, he should have witnessed the stem-enclosing technique of the bamboo.

From the analysis and discussion of the results in this study, there are three main styles used in making Taiwanese traditional bamboo chairs: (1) imitations of Ming and Qing dynasty furniture; (2) the structural treatment in stem-enclosing techniques; and (3) demonstration of the conveniences for everyday life. It displays the wisdom gained from life experiences, and the practical functions of the farming and fishing villages at that time, along with the heritage of daily amenities and the living aesthetics of Fujian and Cantonese cultures.

\section{Conclusions and Suggestions}

Drawn from the aforementioned analysis, this study deems that traditional Taiwanese bamboo chairs can be categorized into eight different types: bamboo stools, side chairs, reclining chairs, armchairs, couple's chairs, double seaters, children's chairs, and others. From the analysis of the contents of the documents of various case studies, it is inferred that there are three major styles in Taiwanese traditional bamboo chairs, namely: (1) imitations of Ming and Qing dynasty furniture; (2) the structural treatment techniques with stem-enclosing; and (3) the display of the simplicity and convenience of pastoral life in Taiwan.

During the process of this study, suggestions such as the followings are to be made: (1) the inheritance and innovation in traditional Taiwanese bamboo furniture; and (2) the originating time and the generation of stem-enclosing techniques. It is hoped that this study can help the Taiwanese to understand traditional Taiwanese bamboo furniture, while acting as a reference to cultural and creative industries, and thus facilitating the re-development of the bamboo art industry.

\section{References}

Bovea, M. D., \& Vidal, R. (2004). Materials selection for sustainable product design: A case study of wood based furniture eco-design. Materials and Design, 25, 111-116.

Chen, C. H., \& Cheng, Y. P. (2005). A study on side and arm chairs of Ming Dynasty using the theory of style. Journal of Design, 10(4), 87-106.

Chung, K. F., \& Yu, W. K. (2002). Mechanical properties of structural bamboo for bamboo scaffoldings. Engineering Structures, 24, 429-442.

Collins, H. (2003). Advanced learner's English dictionary. Glasgow, UK: Harper Collins Publishers. 
Ehrenfeld, J. R. (1997). Industrial ecology: A framework for product and process design. Journal of Cleaner Product, 5(1-2), 87-95.

Ghavami, K. (2005). Bamboo as reinforcement in structural concrete elements. Cement and Concrete Composites, 27, 637-649.

Hornby, A. S. (Ed.). (1996). Oxford advanced learner's English-Chinese dictionary. Taipei: Caves Books.

Lee, P. (2000). 20th century Taiwanese bamboo crafts. Taipei: Artvenue.

Lin, X. (2002). The making graphical essentials of Bamboo furniture. Nantou, Taiwan: National Taiwan Craft Research and Development Institute.

Ross, S. D. (1982). A theory of art. N.Y.: State University of New York.

West, J. A., \& Louys, J. (2007). Differentiating bamboo from stone tool cut marks in the zooarchaeological record, with a discussion on the use of bamboo knives. Journal of Archaeological Science, 34, 512-518.

Wu, S. H., Cheng, Y. P., \& Chen, C. H. (2004). A comparative study between traditional craft and modern design on Taiwanese bamboo furniture. Industrial Design, 32(2), 202-207.

Wu, S. H., Cheng, Y. P., \& Chen, C. H. (2005). A comparative study between traditional makings of bamboo furniture and wood makings (Paper Presented at 10th Conference of Chinese Institute Design: Taiwan).

Yusuf, S., \& Nabeshima, K. (2005). Creative industries in East Asia. Cities, 22(2), 109-122. 